\title{
THE OPTICAL ORBITAL LIGHT CURVE OF THE LOW-MASS X-RAY BINARY V1408 AQUILAE $(=4$ U 1957+115)
}

\author{
Amanda J. Bayless ${ }^{1,2}$, Edward L. Robinson ${ }^{1}$, Paul A. Mason ${ }^{3,4}$, And Paul Robertson ${ }^{1}$ \\ ${ }^{1}$ Department of Astronomy, University of Texas at Austin, 1 University Station, C1400, Austin, TX 78712, USA \\ ${ }^{2}$ Space Science and Engineering Division, Southwest Research Institute, 6220 Culebra Road, San Antonio, TX 78228, USA \\ ${ }^{3}$ Department of Physics, University of Texas at El Paso, El Paso, TX 79968, USA \\ ${ }^{4}$ Department of Mathematics and Physical Science, New Mexico State University-DACC, Las Cruces, NM 88003, USA \\ Received 2010 March 10; accepted 2011 January 6; published 2011 March 3
}

\begin{abstract}
V1408 Aql (= 4U 1957+115) is a low-mass X-ray binary with an orbital period of nearly $9.3 \mathrm{hr}$, whose compact star is a black hole candidate. The system shows a large-amplitude orbital photometric modulation at optical wavelengths. We have obtained new optical photometry of V1408 Aql from which we derive the orbital light curve and an improved orbital ephemeris. We show that the orbital light curve can be reproduced by a model in which the accretion disk around the compact star is thin, axisymmetric, and uneclipsed. The secondary star is heated by $\mathrm{X}$-rays from the compact star and the accretion disk. The orbital modulation is produced entirely by the changing aspect of the irradiated secondary star with orbital phase. Because the system does not eclipse, the fits of the model light curves are insensitive to the orbital parameters, allowing a wide range of orbital inclinations and mass ratios.
\end{abstract}

Key words: accretion, accretion disks - stars: neutron - X-rays: binaries - X-rays: individual (4U 1957+115)

Online-only material: color figures

\section{INTRODUCTION}

Low-mass X-ray binaries (LMXBs) are close binary stars containing a black hole or neutron star surrounded by an accretion disk fueled by mass transferred from a low-mass companion. V1408 Aql is an LMXB with an orbital period of $P=9.329 \pm 0.011 \mathrm{hr}$, derived by Thorstensen (1987) from a roughly sinusoidal modulation he found in its optical light curve. Thorstensen (1987) attributed the modulation to the varying aspect of the secondary star, which is heated by flux from the primary star and its accretion disk. Hakala et al. (1999) obtained two nights of $U B V R I$ photometry, covering one orbital period in total, and also saw an orbital variation, but the light curve was more asymmetric than sinusoidal. Noting that V1408 Aql became bluer when fainter, Hakala et al. (1999) interpreted the minimum in the light curve as a grazing eclipse of a cool, red rim on the accretion disk by the secondary star. In their model the ratio of the rim height to disk radius is large, $H / R \sim 0.2-0.5$, and the orbital modulation outside eclipse is caused by variations in the height of the rim, which obscures more or less of the brighter inner disk. Because it invokes an eclipse, the model implies a high orbital inclination, the best-fitting model having an inclination near $75^{\circ}$.

$4 \mathrm{U} 1957+115$, the X-ray counterpart of V1408 Aql, is a persistent $X$-ray source, showing neither large transient increases in brightness nor faint quiescent states. Its X-ray flux does, however, vary on all timescales from $10 \mathrm{~Hz}(1 \%-2 \%$ rms amplitude) to years (by a factor of four; Wijnands et al. 2002; Russell et al. 2010). The orbital period has not been detected at X-ray wavelengths but Levine \& Corbet (2006) reported a "marginal detection" of a periodicity at $9.3175 \pm 0.0005 \mathrm{hr}$, perhaps related to the orbital period; and Nowak \& Wilms (1999) reported a 117 day period in the $\mathrm{X}$-ray light curve, although this period appears to be evanescent (Wijnands et al. 2002). The distance to $4 \mathrm{U} 1957+115$ is unknown and its mean X-ray luminosity is consequently uncertain; but for a distance of $7 \mathrm{kpc}$ the mean unabsorbed luminosity is $\log \left(L_{\mathrm{X}}\right) \approx 36.5\left(\mathrm{erg} \mathrm{s}^{-1}, 1.5-12 \mathrm{keV}\right)$, while for a distance of $40 \mathrm{kpc}$ it is $\log \left(L_{\mathrm{X}}\right) \approx 38.1$ (Russell et al. 2010).

The X-ray spectrum of $4 \mathrm{U} 1957+115$ has been measured numerous times. It is unusually soft, placing $4 \mathrm{U} 1957+115$ in the region of the X-ray color-color diagram occupied by black hole binaries, including black hole soft X-ray transients (SXTs) in their outburst states, leading to the suspicion that 4U 1957+115 harbors a black hole (White \& Marshall 1984; Schulz et al. 1989; McClintock \& Remillard 2003). If it does contain a black hole, it would be the only known black hole binary in the Galaxy that has not been seen to cycle between low and outburst states. The nearly featureless spectrum can be successfully fitted with a variety of models that include some combination of three components: a soft component from a single- or multi-temperature blackbody, presumably coming from the accretion disk; a hard component that is either a power law, with or without a cutoff, or a thermal Comptonization spectrum; and a broad emission feature near $6.5 \mathrm{keV}$ from $\mathrm{Fe} \mathrm{K}$-line emission (Yaqoob et al. 1993; Singh et al. 1994; Ricci et al. 1995; Nowak \& Wilms 1999; Wijnands et al. 2002). The spectrum is softer when $4 \mathrm{U} 1957+115$ is fainter, harder when it is brighter.

Most recently, Nowak et al. (2008) fit high-quality Chandra, XMM-Newton, and RXTE observations of 4U 1957+115 with diskbb (Mitsuda et al. 1984) and kerrbb models (see Li et al. 2005; Davis et al. 2006) in the XSPEC package, fixing the inclination at $75^{\circ}$, and found that these black hole models fit the observed X-ray spectrum well. The fits required high inner-disk temperatures, $k T_{\text {in }}=1.2-1.8 \mathrm{keV}$, and a low normalization factor, $\left(R_{\mathrm{in}} / D_{10}\right)^{2} \cos i=5-25$, where $R_{\mathrm{in}}$ is the inner radius of the disk in $\mathrm{km}, D_{10}$ is the distance in units of $10 \mathrm{kpc}$, and $i$ is the orbital inclination. There are degeneracies among the derived values for the mass of the compact object, the mass accretion rate, and the distance, but the best-fitting models ranged from a $3 M_{\odot}$ black hole with a spin of $a^{*}>0.82$ at a distance of $10 \mathrm{kpc}$ to a $16 M_{\odot}$ black hole with $a^{*} \approx 1$ at a distance of $22 \mathrm{kpc}$. Nevertheless, the question remains: Is the compact 


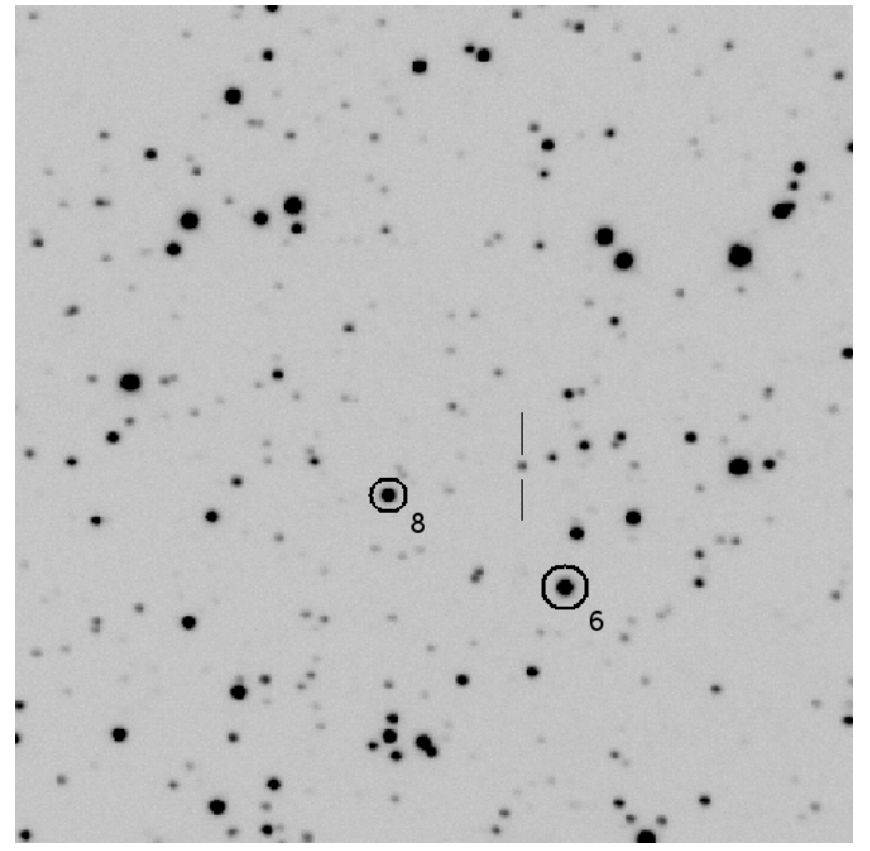

Figure 1. BVR image of V1408 Aql and its surrounding field. V1408 Aql is identified with vertical hash marks and the two comparison stars are circled. The image dimensions are $2^{\prime} .8 \times 22^{\prime} .8$ with north up and east to the left.

object in 4 U $1957+115$ really a black hole? The evidence for a black hole is hardly conclusive. A soft spectrum does not necessarily require a black hole-many SXTs contain neutron stars (Tanaka \& Shibazaki 1996), and Nowak et al. (2008) did not explore models for disks around neutron stars. Furthermore, Yaqoob et al. (1993) argued that the high values of $k T_{\text {in }}$ and low values of $R_{\mathrm{in}}^{2} \cos i$ deduced from fits to the X-ray spectral energy distribution are more typical of neutron star systems than black hole systems.

In this paper, we present new optical photometry of V1408 Aql. We refine the orbital period and derive the mean orbital light curve. There are no eclipses in the light curve. We achieve good fits to the orbital light curve with a model similar to that proposed by Thorstensen (1987) in which the orbital modulation is due to the varying aspect of the irradiated face of the secondary star. We believe this model is more physically realistic than models that invoke a tall disk rim to produce the orbital modulation, but it increases the range of possible orbital inclinations and mass ratios.

\section{OBSERVATIONS AND DATA REDUCTION}

We observed V1408 Aql from 2008 June 2 to June 7 UTC and again from August 2 to August 7 with the Argos prime-focus high-speed CCD photometer on the $2.1 \mathrm{~m}$ Otto Struve telescope at McDonald Observatory (Nather \& Mukadam 2004). We did not obtain data on June 6 or on August 3 and 4 because of clouds. The observations were made through a filter equivalent to a combined $B V R$ filter transmitting the wavelength range 4130-7385 $\AA$ and with an exposure time of 10 s for all frames. The data were reduced using a combination of IRAF routines and an IDL pipeline (R. Hynes 2008, private communication). Data reduction for the Argos photometer is discussed in detail in Hynes et al. (2004). We measured relative fluxes by dividing the flux from V1408 Aql by the total flux from two nearby comparison stars in the frame. Figure 1 shows a stacked image of three $B V R$ images of the field of V1408 Aql for a 30 s effective exposure. V1408 Aql is indicated with vertical hash marks and the two comparison stars are circled. These are the comparison

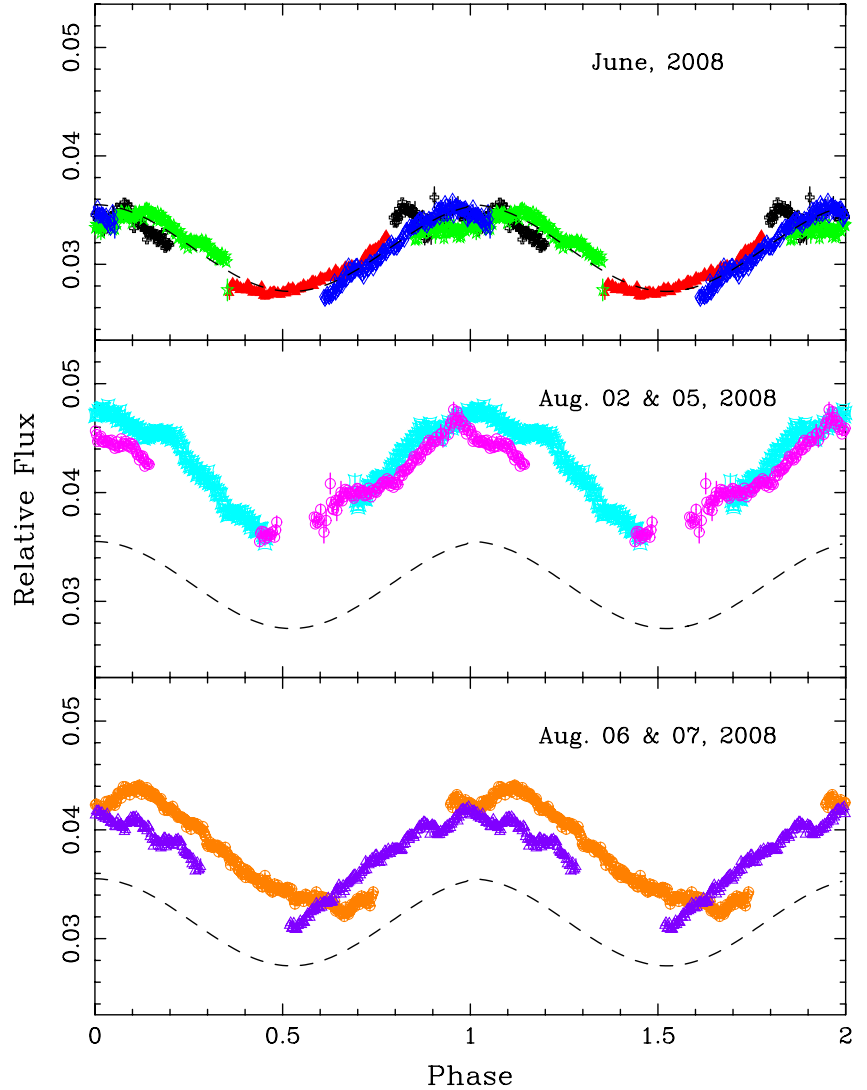

Figure 2. Binned light curves of V1408 Aql. Each color and symbol combination indicate an individual night. Top panel: the fainter state from 2008 June 2 to 7. Middle panel: brighter state from 2008 August 2 to 5. Bottom panel: transition from the brighter state to fainter state on 2008 August 6 and 7. The dashed line is a sine curve fitted to the June data (top panel) and plotted in the middle and bottom panels for comparison. Because V1408 Aql was in transition on August 6 and 7, the orbital modulation is distorted, causing a spurious shift in the orbital phase of maximum light.

(A color version of this figure is available in the online journal.)

stars numbered 6 and 8 in Doxsey et al. (1977) and Margon et al. (1978).

\section{THE OPTICAL LIGHT CURVE AND THE ORBITAL PERIOD}

To search for orbital variations, we folded the light curve on a linear ephemeris with a period of $9.331 \mathrm{hr}$ (see below). Figure 2 shows the phase-folded data from each night binned into 350 equal-width phase bins. The error bars shown in the figure are based on photon counting noise and do not include other noise sources, such as accretion disk flickering. Observations with errors greater than \pm 0.001 in relative flux $(\sim 2.5 \%)$ due to poor seeing or clouds have been discarded. The light curve has a pronounced, roughly sinusoidal orbital modulation. There are also flickering variations with an amplitude of $\sim 5 \%$ of the median flux over an interval of $\sim 0.15$ in phase or $\sim 1.4 \mathrm{hr}$. There are no obvious eclipses.

The mean flux from V1408 Aql changes on timescales of days to months. The system was fainter in June, brighter during the first two observations in August, and fading on August 6 and 7. The top panel of Figure 2 shows the fainter light curve, the middle panel shows the brighter light curve, and the bottom panel shows the transition light curves. The color and symbol combinations indicate the different nights of observations. The dashed line is a sine curve fitted to the June data (top panel) and 




Figure 3. Lomb-Scargle periodogram of the V1408 Aql optical data. The multiple peaks are all two-month aliases of each other introduced by the two-month separation of our observing runs. The small circle and error bar mark the $9.329 \pm$ $0.011 \mathrm{hr}$ period from Thorstensen (1987). While the highest peak is at a frequency of $2.5552 \mathrm{day}^{-1}$, the peak at $2.5722 \pm 0.0001 \mathrm{day}^{-1}$ or $9.331 \pm 0.003 \mathrm{hr}$ agrees with the Thorstensen (1987) period to within a standard deviation. It is this period that we adopt as the orbital period.

plotted in the middle and bottom panels for comparison. The brighter state has $\sim 30 \%$ more flux than the fainter state but the peak-to-valley amplitude of the sinusoidal variations is nearly the same fraction of the median flux in both states.

The orbital light curve of V1408 Aql in 2008 was nearly identical to the light curve measured by Thorstensen (1987) in 1985. In both years the variations of the mean light curve were nearly sinusoidal with, as we will show in Section 5.2, peak-topeak amplitudes near $23 \%$. While it is possible that the mean orbital light curve was truly different in 1996 when observed by Hakala et al. (1999), they constructed the 1996 light curve from data that were obtained on just two nights and covered slightly less than one full orbit with no phase overlap. It is more likely that the light curve was distorted by flickering and night-to-night variations of the mean flux, and is not a good representation of the mean orbital light curve. Since the variations of color with orbital phase are also affected by these problems, it is premature to conclude that V1408 Aql becomes bluer at fainter orbital phases. On the other hand, Figures 2 and 3 in Hakala et al. (1999) do show that any color changes are small and, in the $B V R$ passbands, consistent with zero.

Russell et al. (2010) did not detect the orbital modulation at all in photometry of V1408 Aql obtained between 2006 and 2009, and concluded that the system had changed. Their sparse data set (e.g., 35 data points in $V$ spread over $3.1 \mathrm{yr}$ ) is not, however, well suited for detecting an orbital modulation that has been diluted by flickering and night-to-night variations. Also, their densest data set, from 2008, by chance covers only half the orbit with consequent loss of sensitivity to orbital modulations. Our data, taken within a few weeks of theirs in 2008, show that the orbital modulation was clearly present. The Russell et al. (2010) data do show that the night-to-night variations can have a total range of at least $0.8 \mathrm{mag}$, somewhat higher than the 0.6 mag we observed.

To refine the orbital period we first separately calculated the medians of the fainter state and brighter state light curves and then subtracted the medians to place the faint and bright light curves at zero median flux. We then performed a Lomb-Scargle (L-S) period search, excluding the transition light curves (Lomb 1976; Scargle 1982). The L-S periodogram in Figure 3 shows



Figure 4. One-day-averaged X-ray light curve from RXTE ASM. The times covered to the optical observations in Figure 2 are indicated with the vertical lines.

a series of peaks near the orbital period found by Thorstensen (1987), with the two highest peaks at $9.331 \pm 0.003 \mathrm{hr}$ and $9.393 \pm 0.003 \mathrm{hr}$. All these peaks have vanishingly small false alarm probabilities $\left(\ll 10^{-10}\right)$, reflecting the large amplitude of the orbital variation, and all are two-month aliases of each other introduced by the two-month separation of our observing runs. Because Thorstensen's data were taken over a single 12 day interval and are not subject to one- or two-month aliases, we have used his period to choose among the aliases in our data. His period, $9.329 \pm 0.011 \mathrm{hr}$, agrees with the $9.331 \pm 0.003 \mathrm{hr}$ peak in our L-S periodogram to within a standard deviation and differs from the other peaks in Figure 3 by six or more standard deviations. The orbital ephemeris is, then,

$$
T=2454621.86(02)+0.38877(13) E,
$$

where $T(E=0)$ is the time of maximum flux. We searched for, but did not find, any periodic modulations at other frequencies in the optical data, in agreement with Thorstensen (1987).

\section{THE X-RAY LIGHT CURVE AND X-RAY PERIODICITY SEARCH}

Figure 4 shows the one-day-averaged X-ray light curve of 4U 1957+115 from the RXTE All-Sky Monitor (ASM) ${ }^{5}$ during 2008 May to August. The times of our optical observations are also shown in the figure. The X-ray flux was fairly constant in June when V1408 Aql was faint at optical wavelengths. The $\mathrm{X}$-ray flux was generally higher in late July and August, but on those dates in August when we observed V1408 Aql, the X-ray flux was not any higher than it was in June.

We searched for periodicities in the X-ray light curve from 1996 January to 2008 October in dwell-by-dwell samples using both a least-squares sine curve periodogram and an L-S periodogram. The L-S periodograms in Figures 5 and 6 are fully generalized L-S periodograms (Zechmeister \& Kürster 2009). This method is an improvement over the original L-S periodogram because it includes the weights of individual data points and allows for non-zero means. The periodograms have been normalized assuming that the noise is white. Figures 5 and 6 also show least-squares sine fit periodograms for comparison.

\footnotetext{
5 Data provided by the ASM/RXTE teams at MIT and at the RXTE SOF and GOF at NASA's GSFC, http://xte.mit.edu.
} 


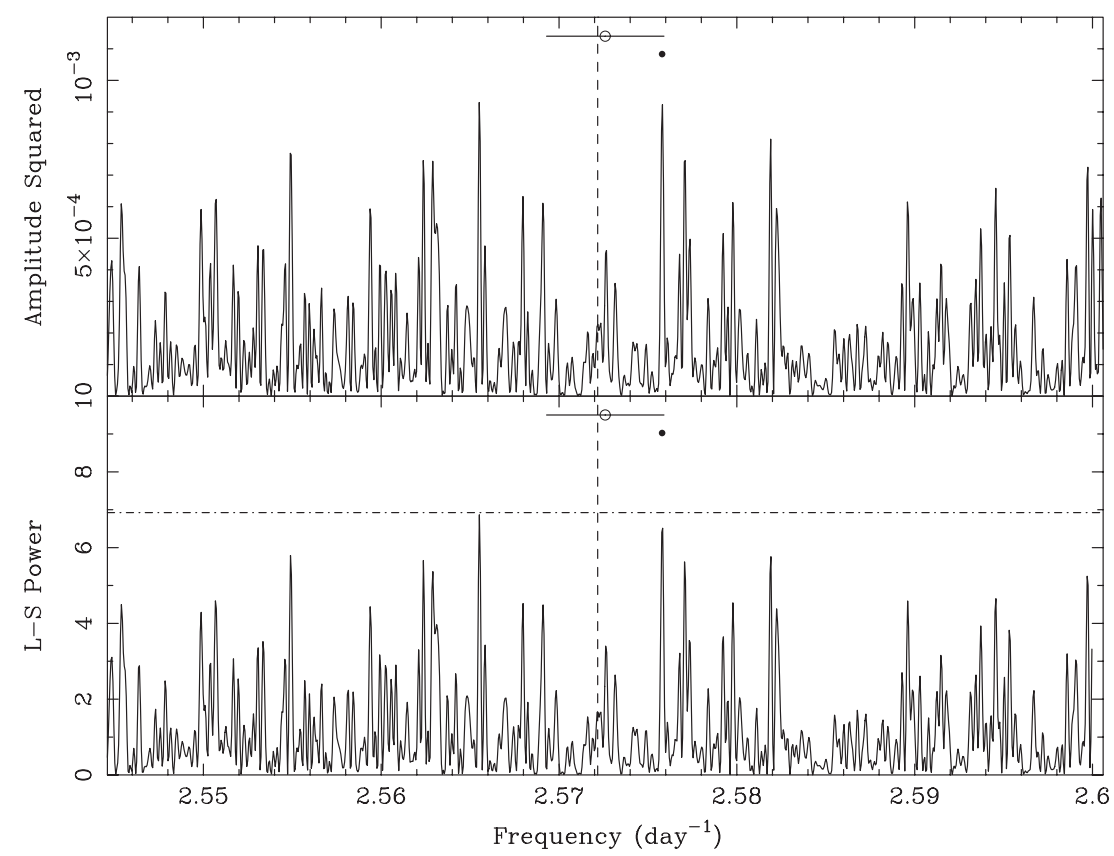

Figure 5. Weighted sine curve (top) and weighted Lomb-Scargle (bottom) periodograms of the RXTE/ASM X-ray light curve of 4U 1957+115. The open circle with error bars marks the orbital frequency determined by Thorstensen (1987) and the vertical dashed line marks our refinement of the orbital period (Equation (1)). The dot marks the "marginal detection" at $P=9.3175 \mathrm{hr}$ or 2.5758 day $^{-1}$ found by Levine \& Corbet (2006). The horizontal dot-dashed line in the bottom panel indicates the $99 \%$ false alarm probability. All of the peaks are consistent with noise.



Figure 6. Low-frequency portion of the weighted sine curve (top) and weighted Lomb-Scargle (bottom) periodograms of the RXTE/ASM X-ray light curve of 4U $1957+115$.

Figure 5 shows the periodograms of the X-ray light curve at periods near the orbital period. The open circle with error bars marks a frequency of $2.5726 \mathrm{day}^{-1}$, corresponding to the orbital period from Thorstensen (1987), and the dashed line marks a frequency of $2.5722 \mathrm{day}^{-1}$, corresponding to the $9.331 \mathrm{hr}$ orbital period from our data. The horizontal dashed line is drawn at the L-S power level for which the false alarm probability is $99 \%$. The dot at 2.5758 day $^{-1}$ marks the "marginal detection" at $P=9.3175 \mathrm{hr}$ from Levine \& Corbet (2006). There are no significant peaks in the periodogram at this or any other frequency between 2.54 and 2.6 day $^{-1}$. Thus, there is no evidence for any modulation of the X-ray flux at periods near the orbital period. Figure 6 shows the low-frequency periodograms. The extra power at frequencies less than $\sim 0.01 \mathrm{day}^{-1}$ is real in the sense that the probability it could be produced by white noise is $\ll 10^{-8}$. None of the individual low-frequency peaks correspond to any of the long periods mentioned by Nowak \& Wilms (1999) and Wijnands et al. (2002), including those at 58.5 day, 117 day, and 234 day. We therefore attribute this excess power to nonperiodic or perhaps transient periodic variations of the X-ray flux. 


\section{A MODEL FOR THE ORBITAL LIGHT CURVE}

\subsection{Preliminary Considerations}

We modeled the orbital light curve of V1408 Aql using our light curve synthesis program ${ }^{6}$ for X-ray binaries (Bayless et al. 2010). The program assumes that the orbital eccentricity is zero, the primary star is a point source surrounded by an accretion disk, and the secondary star fills its Roche lobe. The accretion disk can have a complicated geometry. It can be non-circular, non-axisymmetric, and spotted, and can have structures that extend vertically out of the plane of the disk, such as an interior torus and a tall disk rim. The program uses stellar atmospheres from Kurucz (1996) for the spectrum of the secondary star and blackbody spectra for all other structures. It allows for heating by irradiation but not for second-order heating by the irradiated surfaces. Light curves can be calculated for Johnson/Cousins filters or for a square bandpass over a specified wavelength range. Because we observed with a broadband $B V R$ filter, we used the square bandpass option with wavelength range 4130-7385 A.

The X-ray spectrum of $4 \mathrm{U} 1957+115$ is remarkably simple. Nowak et al. (2008) suggest that it "...may be the cleanest disk spectrum with which to study modern disk models." As noted in the introduction, the continuum can be modeled with just a multi-temperature blackbody disk, perhaps with a weak optically thin Comptonizing corona. With the possible exception of the Fe K-line, there are no strong emission lines. The spectrum does have absorption lines, but Nowak et al. (2008) attribute the lines to absorption in hot-phase interstellar material in the line of sight to $4 \mathrm{U} 1957+115$. The X-ray light curve shows no periodicities that might be attributable to disk warping, superhumps, precession, or other disk asymmetries. Thus, neither the X-ray spectrum nor the X-ray light curve requires models with complex disk structures. We are also loathe to invoke a tall variable-height rim at the outer edge of the disk to modulate the optical orbital light curve. As we have noted previously (Bayless et al. 2010), tall rims are highly unphysical, requiring either supersonic turbulence or temperatures much higher than expected near the edge of the disk.

We have, therefore, adopted a thin, axisymmetric disk in our model. Since there are no eclipses, the only effect of the disk is to add constant flux to the light curve and to irradiate the secondary star, and the only source of orbital modulation is the varying aspect of the irradiated secondary star. This simple model has just five meaningful parameters: the mass ratio, the orbital inclination, the zero point in orbital phase, the X-ray flux from the central source, which heats the secondary star, and an additional constant optical flux from the disk, which also heats the secondary star.

\subsection{Fits to the Light Curve}

A preliminary analysis showed that the model yields synthetic light curves that fit the observed orbital light curves equally well for a wide range of parameters. We therefore abandoned any attempt to define "best" values for the mass ratio and orbital inclination and, instead, explored the range of parameters that yield acceptable fits. We considered three mass ratios, $q=M_{2} / M_{\mathrm{X}}=0.3,0.1$, and 0.025 , roughly corresponding to a compact object that is a neutron star, a low-mass black hole, and a high-mass black hole. For each mass ratio, we fit synthetic light

\footnotetext{
6 A full description of the program is available at http://pisces.as.utexas.edu/robinson/XRbinary.pdf.
}

curves for models with inclinations of $10^{\circ}, 20^{\circ}, 50^{\circ}, 65^{\circ}$, and $70^{\circ}$, plus some for models at $5^{\circ}, 75^{\circ}$, and $80^{\circ}$. For concreteness, we set the mass of the secondary star to $M_{2}=0.4 M_{\odot}$ and its temperature to $3500 \mathrm{~K}$. Our results are nearly independent of $M_{2}$ since the geometry of the system depends only on $q$ and the scale of the system depends only on $M_{2}^{1 / 3}$. The secondary's low temperature prevents its unirradiated surfaces from adding significant flux to the light curve.

We quantified the quality of the fits with $\chi^{2}$ and used the simplex algorithm (Nelder \& Mead 1965) in IDL to find the values of the remaining three parameters (zero phase, X-ray flux, and optical flux) that minimized $\chi^{2}$. Because of the flickering, light curves from individual nights often depart from the mean orbital light curve by much more than the standard deviation of the measurements, which greatly increases $\chi^{2}$. Since the flickering in effect adds non-white noise to the data and the light curves are sampled with a high cadence, the departures are strongly correlated, thwarting our attempts to artificially increase the variances of the individual data points to account for the flickering. As a result, the absolute value of $\chi^{2}$ loses its meaning. Nevertheless, the relative value of $\chi^{2}$ retains its utility for measuring the relative quality of fits.

Figures 7 and 8 show the observed light curves overlaid by fitted synthetic light curves. The figures show fits to the light curves during both the fainter and brighter states, for two inclinations near the limits of the range we explored, $i=20^{\circ}$ and $i=65^{\circ}$, and for the largest and smallest mass ratios we explored, $q=0.3$ (Figure 7) and $q=0.025$ (Figure 8). The peak-to-peak amplitude of the fitted synthetic light curve is $23 \%$ of the mean flux when V1408 Aql is in its fainter state and 24\% of the mean flux when it is in its brighter state. For comparison, Thorstensen (1987) measured an amplitude of 0.116 mag or a peak-topeak amplitude of $22.6 \%$. The independence of the fractional amplitude from the mean flux is an important constraint. We interpret the constraint to mean that (1) the optical flux from both the disk and the secondary star is dominated by reprocessed Xray flux and (2) the flux-weighted temperatures of the irradiated surfaces are high enough that the emitted optical flux is on the Rayleigh-Jeans tail of the blackbody distribution. Changes in the X-ray flux then produce proportional changes in the optical flux from the disk and from the heated face of the secondary star, leaving their relative contributions to the optical flux unchanged. The blue colors of V1408 Aql $(B-V \approx 0.13, U-B \approx-0.70$, $E(B-V) \approx 0.3$; Margon et al. 1978; Hakala et al. 1999) are at least roughly consistent with this interpretation.

We achieved equally good fits to the orbital light curves for all three mass ratios. The dominant effect of increasing the mass ratio is to increase the relative size of the secondary star, but the effect of the increased size can be offset by reducing the amount of irradiative heating. As a result, the observed light curves do not by themselves yield useful limits on the mass ratio.

We have two ways to place an upper limit on the orbital inclination. The first is from the lack of eclipses in the light curve. Let us assume that the accretion disk extends to its tidal truncation radius, $R_{d}=0.9 R_{L 1}$, where $R_{L 1}$ is the radius of a sphere with the same volume as the Roche lobe around the compact object. Eclipses of the disk would be detected in the mean light curve if they were more than about $2 \%$ deep. This occurs at $i \gtrsim 65^{\circ}$ for $q=0.3$, at $i \gtrsim 70^{\circ}$ for $q=0.1$, and at $i \gtrsim 75^{\circ}$ for $q=0.025$. The limiting inclinations are higher if $R_{d}<0.9 R_{L 1}$. The second way to place an upper limit on the inclination is from a flattening of the minimum of the synthetic light curves at high orbital inclinations. Figure 9 shows synthetic 


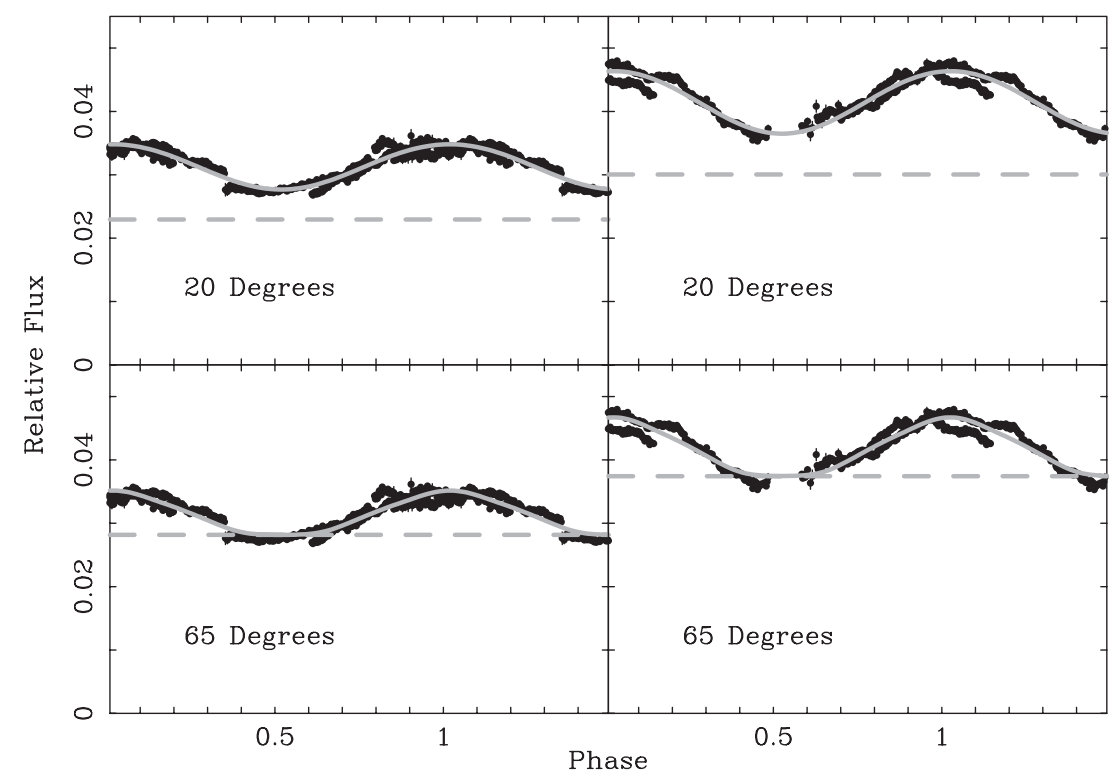

Figure 7. Observed orbital light curves of V1408 Aql in its low state (left panels) and high state (right panels). The observed light curves are overlaid with fitted synthetic light curves calculated for $q=0.3$. The orbital inclinations are $i=20^{\circ}$ (top panels) and $i=65^{\circ}$ (bottom panels). The dashed line shows the contribution from the accretion disk, the remaining flux coming from the heated secondary star.



Figure 8. Same as Figure 7, but for $q=0.025$.

light curves for $q=0.3$ (top, red line), $q=0.1$ (middle, green line), and $q=0.025$ (bottom, blue line), each for an inclination of $70^{\circ}$. The synthetic light curves all flatten at minimum light. This flattening is unrelated to eclipses but instead occurs because less than half the secondary star is irradiated, because the edges of the irradiated region are heated to lower temperatures, and because the secondary is not spherical. The lack of flat minima in the observed optical light curves places an upper limit of $70^{\circ}$ on the orbital inclination. This second limit is independent of the mass ratio and the size of the accretion disk, and is more reliable than the limits set by the lack of disk eclipses.

The synthetic light curves for models with orbital inclinations of $20^{\circ}$ and $65^{\circ}$ both fit the observed light curves well. The fit for $20^{\circ}$ has a slightly smaller $\chi^{2}$ but the difference is not statistically significant. The dominant effect of decreasing the orbital inclination is to decrease the amplitude of the orbital modulation caused by the changing aspect of the secondary star, but the decrease of the relative amplitude can be offset by decreasing the fraction of the flux contributed by the accretion disk. This is shown in Table 1, which gives the fraction of the optical flux, $\alpha$, that comes from the accretion disk as a function of orbital inclination; the fraction is also shown as the dashed lines in Figures 7 and 8. The value of $\alpha$ is nearly independent of the mass ratio and the luminosity state, but decreases with orbital inclination, dropping slowly from $\sim 88 \%$ at $i=65^{\circ}$ to $\sim 67 \%$ at $i=20^{\circ}$, and then dropping rapidly to zero at $\sim 5^{\circ}$.

The flickering in the light curve disfavors inclinations less than $20^{\circ}$. The low values of $\alpha$ at $i<20^{\circ}$ would require an improbably high fraction of the disk flux to be modulated to produce the observed amplitude of the flickering. 


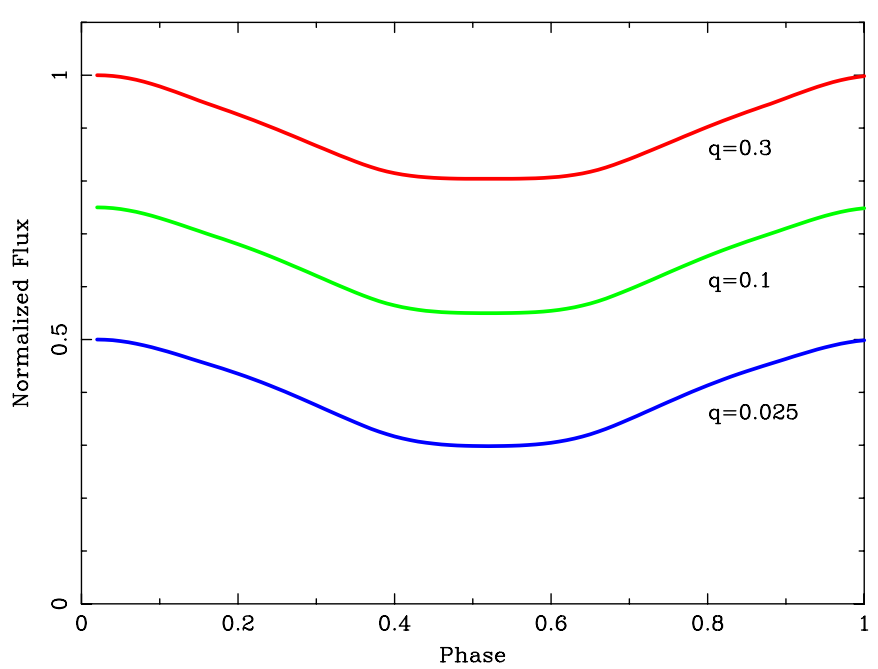

Figure 9. Synthetic light curves fitted to the observed light curves of V1408 Aql in its fainter state. The orbital inclination is $70^{\circ}$ for each light curve, and the mass ratios are $q=0.3$ (top, red line), $q=0.1$ (middle, green line), and $q=0.025$ (bottom, blue line). The models have been normalized to 1.0 at maximum and then offset by 0.25 for clarity. At this and higher orbital inclinations the minimum of the light curve is flat bottomed.

(A color version of this figure is available in the online journal.)

Table 1

Properties of the Fitted Models

\begin{tabular}{lcccccc}
\hline \hline Inclination & \multicolumn{2}{c}{$\begin{array}{c}\text { Fraction of Flux from } \\
\text { Disk }\end{array}$} & & \multicolumn{3}{c}{ Inferred $^{\mathrm{a}} T_{2} / T_{d}$} \\
\cline { 2 - 3 } \cline { 5 - 7 } & Faint State & Bright State & & $q=0.3$ & $q=0.1$ & $q=0.025$ \\
\hline $10^{\circ}$ & 0.337 & 0.334 & & 9.49 & 24.9 & 78.0 \\
$20^{\circ}$ & 0.683 & 0.666 & & 2.28 & 5.97 & 18.7 \\
$30^{\circ}$ & 0.784 & 0.759 & & 1.33 & 3.48 & 10.9 \\
$40^{\circ}$ & 0.830 & 0.808 & & 0.88 & 2.31 & 7.23 \\
$50^{\circ}$ & 0.857 & 0.839 & & 0.60 & 1.56 & 4.90 \\
$65^{\circ}$ & 0.880 & 0.876 & & 0.29 & 0.76 & 2.38 \\
$70^{\circ}$ & 0.884 & 0.869 & & 0.25 & 0.65 & 2.05 \\
\hline
\end{tabular}

Note. ${ }^{\text {a }} T_{2} / T_{d}$ is the temperature ratio required to produce the observed amplitude of the orbital modulation assuming $R_{d}=0.9 R_{L 1}$.

\subsection{Constraints on $q$ and $i$ from Color and Temperature}

The results from the previous section show that neither the mass ratio nor the orbital inclination are strongly constrained by the shape of the light curve. They are, however, constrained by color and temperature, albeit rather weakly and with some uncertainty. We take as our starting point that the optical flux from V1408 Aql is dominated by regions of the secondary star and disk that are on the Rayleigh-Jeans tail of the blackbody distribution. Let $T_{2}$ and $T_{d}$ be the flux-weighted temperatures of the irradiated areas of the secondary star and disk so that $T_{2} / T_{d}$ is the ratio of their emitted optical fluxes. We have two ways to calculate $T_{2} / T_{d}$. The first is from the amplitude of the orbital modulation, which is nearly independent of the luminosity and linear scale of the model, and the second is from the irradiative heating of the disk and secondary star, which depends strongly on the luminosity and scale of the model. The two calculations must agree for the model to be internally consistent.

From the amplitude of the orbital modulation, the ratio of the optical energy coming from the secondary to the optical energy
Table 2

Typical Temperature of the Irradiated Surface of the Secondary Star

\begin{tabular}{lcc}
\hline \hline Mass Ratio & \multicolumn{2}{c}{ Temperature (K) } \\
\cline { 2 - 3 } & $L_{\mathrm{X}}=10^{37} \mathrm{erg} \mathrm{s}^{-1}$ & $L_{\mathrm{X}}=10^{38} \mathrm{erg} \mathrm{s}^{-1}$ \\
\hline 0.3 & 28,000 & 50,000 \\
0.1 & 22,000 & 40,000 \\
0.025 & 17,000 & 31,000 \\
\hline
\end{tabular}

from the disk is

$$
\left(\frac{T_{2}}{T_{d}}\right)\left(\frac{A_{2} / 2}{A_{d} \cos i}\right) \approx \frac{1-\alpha}{\alpha},
$$

where $A_{2} / 2$ and $A_{d} \cos i$ are the areas of the secondary star and disk that contribute significantly to the optical flux, the factor of two because only half the secondary is irradiated and the factor of $\cos i$ because the disk is foreshortened. If the disk has expanded to its tidal truncation radius, then $R_{d}=0.9 R_{L 1}$, and Equation (2) can be rewritten as

$$
\frac{T_{2}}{T_{d}}=\frac{1-\alpha}{\alpha}\left[\frac{0.81\left(R_{L 1} / a\right)^{2} \cos i}{0.5\left(R_{L 2} / a\right)^{2}}\right],
$$

where $a$ is the semi-major axis, and $R_{L 1} / a$ and $R_{L 2} / a$ are functions of $q$ and can be calculated from, for example, Eggleton's approximation (Eggleton 1983). Table 1 gives the values of $T_{2} / T_{d}$ implied by the fits to the light curves. Note that $T_{2} / T_{d}$ must be larger for smaller mass ratios because the relative size of the secondary star is smaller.

The temperature of the irradiated secondary depends primarily on $q$, the scale of the system (set by $M_{2}$ ), the X-ray luminosity, and the fraction of the incident radiation that is locally thermalized and re-emitted. Table 2 gives typical values for $T_{2}$ for X-ray luminosities of $10^{37}$ and $10^{38} \mathrm{erg} \mathrm{s}^{-1}$ for each of the three mass ratios, where we have assumed that the X-ray energy is produced near the compact object and that all the X-ray energy incident on the secondary star is thermalized and re-emitted. The temperature of an irradiated disk is extremely sensitive to disk geometry and the location of the source of the irradiating X-rays (see, e.g., Hynes 2005). As a result, our models give no reliable constraint on $T_{d}$. We will simply take that $T_{d}$ must be greater than $\sim 10^{4} \mathrm{~K}$ for its optical flux to be on the Rayleigh-Jeans tail of the blackbody distribution. It is possible, of course, that $T_{d}$ is substantially greater than $10^{4} \mathrm{~K}$.

We can now calculate $T_{2} / T_{d}$ the second way, using $T_{2}$ from Table 2 and taking $T_{d}>10^{4} \mathrm{~K}$ so that the resulting $T_{2} / T_{d}$ is an upper limit. For $q=0.3$, the upper limits are $T_{2} / T_{d}<5$ for $L_{\mathrm{X}}=10^{38} \mathrm{erg} \mathrm{s}^{-1}$ and $T_{2} / T_{d}<2.8$ for $L_{\mathrm{X}}=10^{37} \mathrm{erg} \mathrm{s}^{-1}$ These values are consistent with the values in Table 1 for any $i \gtrsim 20^{\circ}$ and yield no additional useful constraint on the inclination. For $q=0.1$, the upper limits are 4.0 and 2.2 and are mildly constrained, needing $i \gtrsim 30^{\circ}$ for $10^{38} \mathrm{erg} \mathrm{s}^{-1}$ and $i \gtrsim 40^{\circ}$ for $10^{37} \mathrm{erg} \mathrm{s}^{-1}$. At $q=0.025$, the upper limits are 3.1 and 1.7 and become more strongly constraining. Only the model with $L_{\mathrm{X}}=10^{38} \mathrm{erg} \mathrm{s}^{-1}$ is internally consistent and even it requires $i \gtrsim 60^{\circ}$.

These constraints are not, however, as certain as one might wish. The values of $T_{2} / T_{d}$ given in Table 1 are directly proportional to the adopted area of the accretion disk. If the radius of the accretion disk is as small as the circularization radius, $T_{2} / T_{d}$ is reduced by a factor of four or more, and the inclination becomes essentially unconstrained even for 
$q=0.025$. On the other hand, if $T_{d}$ is greater than $10^{4} \mathrm{~K}$, the constraints on the orbital inclination become more severe. Thus, the most that can be concluded is that higher mass ratios are mildly favored and the models are internally consistent over a wide range of inclinations and mass ratios.

Finally, it is possible that further observations may show that V1408 Aql does, indeed, become bluer at fainter orbital phases as claimed by Hakala et al. (1999). Our model can reproduce such color variations-all that is required is that the heated face of the secondary star have a temperature less than the temperature of the accretion disk. This would strongly constrain the permitted mass ratios and inclinations, eliminating the $q=0.025$ models altogether and restricting the $q=0.1$ models to inclinations greater than $\sim 65^{\circ}$ and the $q=0.3$ models to inclinations greater than $\sim 40^{\circ}$.

\section{SUMMARY AND DISCUSSION}

We obtained high-speed photometry of V1408 Aql on nine nights in June and August of 2008. The optical light curve flickered by at least $5 \%$ on timescales of minutes to hours and the orbit-averaged flux varied by $30 \%$ on timescales of a few days. The mean orbital light curve was roughly sinusoidal with a peak-to-peak amplitude near $23 \%$ and was not measurably different from the light curve observed by Thorstensen (1987) in 1985. We attribute the lack of an orbital modulation reported by Russell et al. (2010) and the different shape of the orbital light curve found by Hakala et al. (1999) to the sparseness of their data sets and to the distortions of the light curve induced by flickering and night-to-night variations. The refined orbital period is $0.38877 \pm 0.00013$ days (Equation (1)). There are no significant periodicities in the RXTE ASM X-ray light curve at or near the orbital period.

The orbital light curve is consistent with a model in which the accretion disk around the compact star is thin, axisymmetric, and uneclipsed. The secondary star is irradiated and heated by $\mathrm{X}$-rays from the compact star and the accretion disk. The orbital modulation is caused entirely by the varying aspect of the heated face of the secondary star with orbital phase. This simple model yields consistent fits for all mean light levels and it avoids an unphysically tall disk rim.

At inclinations greater than $70^{\circ}$, the synthetic orbital light curves flatten at minimum light. The lack of a flat minimum in the observed light curve places an upper limit of $\sim 70^{\circ}$ on the orbital inclination. We cannot place an unimpeachable lower limit on the orbital inclination but argue that both the amplitude of the flickering and the lack of color variations over the orbit imply that the orbital inclination is greater than $20^{\circ}$. Thus, the orbital inclination lies between $20^{\circ}$ and $70^{\circ}$. We achieved good fits to the light curves with mass ratios from 0.025 to 0.3 . If the radius of the accretion disk is close to the tidal truncation radius, the smaller mass ratios, which correspond to systems in which the primary is a black hole, require X-ray luminosities nearer $10^{38} \mathrm{erg} \mathrm{s}^{-1}$ and orbital inclinations in the upper half of the permitted range.

Figure 8 in Russell et al. (2010) plots optical luminosity against X-ray luminosity for V1408 Aql and other LMXBs. The distance to V1408 Aql is unknown, but Russell et al. (2010) adopt three distances for comparison. If the distance is $7 \mathrm{kpc}$, the X-ray luminosity is $\sim 10^{36.5} \mathrm{erg} \mathrm{s}^{-1}$, and at $20 \mathrm{kpc}$, it is $\sim 10^{37.5} \mathrm{erg} \mathrm{s}^{-1}$, placing V1408 Aql in a region of the figure occupied by neutron star binaries but not black hole binaries. At $40 \mathrm{kpc}$, the X-ray luminosity is $\sim 10^{38.5} \mathrm{erg} \mathrm{s}^{-1}$ and V1408 Aql falls in a region occupied by some soft-state black hole binaries and the neutron star $Z$-sources. All these possibilities remain viable with our new model.

If, however, V1408 Aql contains a black hole it would be unusual in two ways. First, as mentioned earlier it would be the only known black hole binary in the Galaxy that has not been seen to cycle between low and outburst states. Second, irradiative heating is more typical of neutron star X-ray binaries than black hole binaries. This is shown most clearly by SXTs during their outbursts. Large-amplitude modulations from irradiative heating have been observed during outbursts of Aql X-1 and XTE 2123-058 and possibly X1608-52, all of which are classical SXTs containing neutron stars, and have also been observed in the related neutron star X-ray transient XTE J2129+47 (Thorstensen et al. 1979; Welsh et al. 2000; Zurita et al. 2000; Wachter et al. 2002). In contrast, orbital modulation from irradiative heating of the secondary star is rarely observed in black hole SXTs and, if it is observed, its amplitude is low (Neil et al. 2007). This is due at least partly to the lower mass ratios of black hole binaries. At lower mass ratios the secondary star is comparatively smaller. As shown in Section 5.3, the smaller secondary can produce a largeamplitude orbital modulation only if its irradiated face is heated to high temperatures.

Finally, the light curves of the black hole SXTs often show superhumps (Haswell et al. 2001; Zurita et al. 2006; Kato et al. 2009). Such superhumps are ubiquitous in mass transfer binaries with disks whose radii cross the 3:1 orbital resonance, which requires a mass ratio less than $\sim 0.25$ (Lubow 1991; Kato et al. 2009). The failure of V1408 Aql to show superhumps in its light curve argues that its mass ratio is larger than $\sim 0.25$ and the mass of its primary star is less than $\sim 1.6 M_{\odot}$. All the known black holes in binary stars have masses greater than about $4 M_{\odot}$ (Casares 2006). While none of these arguments are conclusive, together they weigh heavily in favor of a neutron star and against a black hole in V1408 Aql.

We thank R. I. Hynes for helpful discussions.

\section{REFERENCES}

Bayless, A. J., Robinson, E. L., Hynes, R. I., Ashcraft, T. A., \& Cornell, M. E. 2010, ApJ, 709, 251

Casares, J. 2006, in The Many Scales in the Universe: JENAM 2004 Astrophysics Reviews, ed. J. C. Del Toro Iniesta, E. J. Alfaro, J. G. Gorgas, E. Salvador-Sole, \& H. Butcher (Dordrecht: Springer), 145

Davis, S. W., Done, C., \& Blaes, O. M. 2006, ApJ, 647, 525

Doxsey, R. E., Bradt, H. V., Dower, R. G., Jernigan, J. G., \& Apparao, K. M. V. 1977, Nature, 269, 112

Eggleton, P. P. 1983, ApJ, 268, 368

Hakala, P. J., Muhli, P., \& Dubus, G. 1999, MNRAS, 306, 701

Haswell, C. A., King, A. R., Murray, J. R., \& Charles, P. A. 2001, MNRAS, 321,475

Hynes, R. I. 2005, ApJ, 623, 1026

Hynes, R. I., Robinson, E. L., \& Jeffery, E. 2004, ApJ, 608, L101

Kato, T., et al. 2009, PASJ, 61, 395

Kurucz, R. L. 1996, in ASP Conf. Ser. 108, M.A.S.S.: Model Atmospheres and Spectrum Synthesis, ed. S. J. Adelman, F. Kupka, \& W. W. Weiss (San Francisco, CA: ASP), 2

Levine, A. M., \& Corbet, R. 2006, ATel, 940, 1

Li, L., Zimmerman, E. R., Narayan, R., \& McClintock, J. E. 2005, ApJS, 157, 335

Lomb, N. R. 1976, Ap\&SS, 39, 447

Lubow, S. H. 1991, ApJ, 381, 259

Margon, B., Thorstensen, J. R., \& Bowyer, S. 1978, ApJ, 221, 907

McClintock, J. E., \& Remillard, R. A. 2003, in Compact Stellar X-ray Sources, ed. W. H. G. Lewin \& M. van der Klis (Cambridge: Cambridge Univ. Press), 157 
Mitsuda, K., et al. 1984, PASJ, 36, 741

Nather, R. E., \& Mukadam, A. S. 2004, ApJ, 605, 846

Neil, E. T., Bailyn, C. D., \& Cobb, B. E. 2007, ApJ, 657, 409

Nelder, J. A., \& Mead, R. 1965, Comput. J., 7, 308

Nowak, M. A., Juett, A., Homan, J., Yao, Y., Wilms, J., Schulz, N. S., \& Canizares, C. R. 2008, ApJ, 689, 1199

Nowak, M. A., \& Wilms, J. 1999, ApJ, 522, 476

Ricci, D., Israel, G. L., \& Stella, L. 1995, A\&A, 299, 731

Russell, D. M., Lewis, F., Roche, P., Clark, J. S., Breedt, E., \& Fender, R. P. 2010, MNRAS, 402, 2671

Scargle, J. D. 1982, ApJ, 263, 835

Schulz, N. S., Hasinger, G., \& Truemper, J. 1989, A\&A, 225, 48

Singh, K. P., Apparao, K. M. V., \& Kraft, R. P. 1994, ApJ, 421, 753
Tanaka, Y., \& Shibazaki, N. 1996, ARA\&A, 34, 607

Thorstensen, J., Charles, P., Bowyer, S., Briel, U. G., Doxsey, R. E., Griffiths, R. E., \& Schwartz, D. A. 1979, ApJ, 233, L57

Thorstensen, J. R. 1987, ApJ, 312, 739

Wachter, S., Hoard, D. W., Bailyn, C. D., Corbel, S., \& Kaaret, P. 2002, ApJ, 568,901

Welsh, W. F., Robinson, E. L., \& Young, P. 2000, AJ, 120, 943

White, N. E., \& Marshall, F. E. 1984, ApJ, 281, 354

Wijnands, R., Miller, J. M., \& van der Klis, M. 2002, MNRAS, 331, 60

Yaqoob, T., Ebisawa, K., \& Mitsuda, K. 1993, MNRAS, 264, 411

Zechmeister, M., \& Kürster, M. 2009, A\&A, 496, 577

Zurita, C., et al. 2000, MNRAS, 316, 137

Zurita, C., et al. 2006, ApJ, 644, 432 